\begin{tabular}{|c|l|}
\hline Title & Carbon nanotube coated silicone as a flexible and electrically conductive biomedical material \\
\hline Author(s) & Matsuoka, Makoto; A kasaka, Tsukasa; Totsuka, Y asunori; W atari, Fumio \\
\hline Citation & $\begin{array}{l}\text { Materials Science and Engineering: C, 32(3), 574.580 } \\
\text { https://doi.org/40.1016/.msec.2011.12.011 }\end{array}$ \\
\hline Issue Date & 2012-04_01 \\
\hline Doc URL & http://hdl.handle.net/2115/49007 \\
\hline Type & article(author version) \\
\hline File Information & MSEC32-3_574-580.pdf \\
\hline
\end{tabular}

Instructions for use 


\section{Carbon nanotube-coated silicone as a flexible and electrically conductive biomedical material}

Makoto Matsuoka ${ }^{\mathrm{a}, \mathrm{b},}{ }^{*}$, Tsukasa Akasaka $^{\mathrm{c}}$, Yasunori Totsuka ${ }^{\mathrm{a}}$ and Fumio Watari ${ }^{\mathrm{c}}$

a Department of Oral and Maxillofacial Surgery, Graduate School of Dental Medicine, Hokkaido University, Kita 13 Nishi 7, Kita-ku, Sapporo 060-8586, Japan

${ }^{\mathrm{b}}$ Research Fellow of the Japan Society for the Promotion of Science, Japan

${ }^{\mathrm{c}}$ Department of Dental Materials and Engineering, Graduate School of Dental Medicine, Hokkaido University, Kita 13 Nishi 7, Kita-ku, Sapporo 060-8586, Japan

*Corresponding author. Tel.: +81 11706 4283; Fax: +81 11706 4283; E-mail: matsuoka@den.hokudai.ac.jp 


\begin{abstract}
Artificial cell scaffolds that support cell adhesion, growth, and organization need to be fabricated for various purposes. Recently, there have been increasing reports of cell patterning using electrical fields. We fabricated scaffolds consisting of silicone sheets coated with single-walled (SW) or multi-walled (MW) carbon nanotubes (CNTs) and evaluated their electrical properties and biocompatibility. We also performed cell alignment with dielectrophoresis using CNT-coated sheets as electrodes. Silicone coated with 10 $\mu \mathrm{g} / \mathrm{cm}^{2} \mathrm{SWCNTs}$ exhibited the least sheet resistance $(0.8 \mathrm{k} \Omega / \mathrm{sq})$; its conductivity was maintained even after 100 stretching cycles. CNT coating also improved cell adhesion and proliferation. When an electric field was applied to the cell suspension introduced on the CNT-coated scaffold, the cells became aligned in a pearl-chain pattern. These results indicate that CNT coating not only provides electro-conductivity but also promotes cell adhesion to the silicone scaffold; cells seeded on the scaffold can be organized using electricity. These findings demonstrate that CNT-coated silicone can be useful as a biocompatible scaffold.
\end{abstract}

Keywords: Carbon nanotubes, silicone, scaffold, electro conductive, dielectrophoresis (DEP) 


\section{Introduction}

Tissue engineering aims to create artificial tissue that can be implanted to replace damaged parts of the human body. To achieve this goal, many types of cell scaffolds for tissue engineering have been studied [1,2]. Artificial scaffolds need to be designed to support cell adhesion, growth, and organization. With these aims, scaffolds with a variety of functions have been developed [3-5].

Carbon nanotubes (CNTs) are one particular type of nanomaterial; the walls of each nanotube are derived from a graphene sheet. In general, there are 2 groups of carbon nanotubes: single-walled carbon nanotubes (SWCNTs) and multi-walled carbon nanotubes (MWCNTs). SWCNTs are composed of a single cylindrical graphene sheet, while MWCNTs consist of concentric tubes with multiple layers of carbon walls (Figure 1). Both types of CNTs have attracted increasing attention due to their unique mechanical, electrical, and structural properties [6,7]. Because of their unique properties, they are used in various biomedical fields for a variety of applications, including the manufacturing of biosensors, drug and vaccine delivery vehicles, and materials for tissue engineering [8-10]. Furthermore, an increasing number of reports are focusing on the use of CNTs as scaffolds in regenerative medicine [11-13]. Neuron differentiation from human embryonic stem cells has been reported to be promoted on CNT-based scaffolds [14]. Furthermore, osteoblastic cells have been found to adhere strongly to CNTs $[15,16]$. CNTs are useful as a scaffold material because of their excellent mechanical properties and fiber-like structure, which is close to the size of collagen fibrils (approximately 40-100 $\mathrm{nm}$ in diameter, depending on 
tissue site [17-19]). In addition, one of the most attractive properties of CNTs is their excellent conductivity, which enables them to provide electro-conductivity to a scaffold. Recently, many reports of cell positioning and patterning using electrical fields have been published [20,21]. The dielectrophoretic (DEP) approach is one of the most useful methods because of its ability to move cells in a non-uniform electric field [22-24]. An electroconductive CNT scaffold would enable cell patterning and alignment and would therefore be beneficial for tissue organization.

Silicone is widely used in the field of plastic surgery because of its flexibility and stability inside the body. Although silicone is the favored implant material due to its chemical stability and flexibility, it has limited cell adhesiveness and can therefore cause problems during the course of long-term usage [25-27]. We previously reported improvements in osteoblastic cell (Saos-2) proliferation on silicone rubber as a result of CNT coating [28]. However, in that study, the CNTs on the silicone surface were not firmly fixed using the coating method we used, and therefore, the CNTs subsequently detached. The purpose of this study was to improve the fabrication method of CNT-coated silicone and evaluate its properties and biocompatibility as a flexible, electrically conductive biomedical material.

\section{Material and methods}

\subsection{Substrate preparation}

\subsubsection{Preparation of CNT-coated silicone}


Both SWCNTs and MWCNTs were used in this study. The SWCNTs, synthesized using the arc discharge method, were $0.8-2.5 \mathrm{~nm}$ in diameter and were $>95 \%$ pure (Meijo Nano Carbon Co. Ltd., Nagoya, Japan) [29]. The MWCNTs, synthesized using the chemical vapor deposition technique (NanoLab Inc., MA, USA), were 20-40 nm in diameter and were $98 \%$ pure [30]. Both types of CNTs were dispersed in $99.5 \%$ ethanol by sonication. The resultant dispersion was vacuum filtered using a porous polycarbonate membrane $47-\mathrm{mm}$ in diameter with a $0.2-\mu \mathrm{m}$ pore size (Polycarbonate Membrane Filter; Toyo Roshi Kaisha, Ltd., Tokyo, Japan), and a thin film of CNTs was fabricated on the polycarbonate membrane. Medical-grade silicone elastomer and cross-linker (Platinum Silicone Elastomer, A-2186-F; Factor II, Inc., AZ, USA) were mixed in a ratio of 10:1 by weight and applied in a thin layer on the CNT film. The silicone elastomer was allowed to polymerize at room temperature overnight. After polymerization, the polycarbonate membrane was removed by peeling and the CNTs remained fixed onto the silicone sheet surface. Silicone rubber sheets coated with CNTs at densities of 1.0, 2.5, 5.0, 7.5, or 10 $\mu \mathrm{g} / \mathrm{cm}^{2}$ were prepared using each type of CNT for a total of 10 conditions; silicone sheets without CNTs $\left(0 \mu \mathrm{g} / \mathrm{cm}^{2}\right)$ were also prepared as uncoated silicone controls.

\subsection{Characterization of CNT-coated silicone}

\subsubsection{Structural properties}

The surface morphologies of uncoated silicone and SWCNT- and MWCNT-coated silicone $\left(10 \mu \mathrm{g} / \mathrm{cm}^{2}\right)$ were observed using an atomic force microscope (AFM) 
(MFP-3D-BIO-J; Asylum Technology Co. Ltd.., Tokyo, Japan). The scan size was set at 5 $\mu \mathrm{m}$, and the scan rate was $1 \mathrm{~Hz}$. Section analysis was performed using IGOR Pro 6.12A software (Wave Metrics, Inc., OR, USA).

Surface wettability was assessed by water contact angle measurements. Droplets of distilled water $(2 \mu \mathrm{L})$ on the substrates were photographed horizontally with a digital microscope, and the contact angles were determined using the ImageJ $1.43 \mathrm{e}$ (NIH, USA) LB-ADSA plugin [31]. Three samples were used for the test. The mean values are presented with standard deviations (SD).

\subsubsection{Tape test}

The adhesion of the CNTs to the silicone surfaces was evaluated by the tape test. The test was performed with $10 \mu \mathrm{g} / \mathrm{cm}^{2}$ SWCNT- and MWCNT-coated silicone. CNT-coated surfaces were covered with a strip of adhesive tape (Scotch Tape; Sumitomo 3M Co. Ltd., Tokyo, Japan), which was subsequently peeled off. The optical transparencies of the samples were measured with a luminous transmittance meter (STS-4; Fujikoden, Kanagawa, Japan) before and after tape peeling. The transparency of uncoated silicone was set at $100 \%$. Measurements were performed on 5 samples each, and the results are presented as mean \pm standard deviation (SD). AFM images after the tape test were also obtained. The scan size was set at $5 \mu \mathrm{m}$, and the scan rate was $1 \mathrm{~Hz}$. 


\subsubsection{Sheet resistance}

The sheet resistance of both types of CNT-coated silicone was measured using a four-probe technique with a resistivity meter [32] (MCP-T 610; Mitsubishi Chemical Analytech Co. Ltd., Kanagawa, Japan). To evaluate changes in the resistance due to stretching, each sample was maintained at $20 \%$ uniaxial stretch, and sheet resistance was measured with the probe. The resistance of SWCNT-coated silicone $\left(10 \mu \mathrm{g} / \mathrm{cm}^{2}\right)$ after cyclic strain was also measured; sheet resistance was measured after 0, 1, 20, 40, 60, 80, and 100 cycles of uniaxial stretching. All measurements were performed using 12 samples.

\subsection{Cell culture assay}

\subsubsection{Cell culture}

C2C12 myoblast cells were obtained from DS Pharma Biomedical (ECACC 91021101, Osaka, Japan), and were grown in Dulbecco's modified Eagle's medium (DMEM; Sigma-Aldrich, MO, USA), supplemented with 10\% fetal bovine serum (FBS; MP Biomedicals, Eschwege, Germany) under standard cell culture conditions $\left(37^{\circ} \mathrm{C}\right.$ in a humidified atmosphere of $5 \% \mathrm{CO}_{2}$ in air). The culture medium was replaced every other day. Three types of scaffold were used in the cell culture assays: uncoated silicone, SWCNT-coated silicone $\left(10 \mu \mathrm{g} / \mathrm{cm}^{2}\right)$, and MWCNT-coated silicone $\left(10 \mu \mathrm{g} / \mathrm{cm}^{2}\right)$.

\subsubsection{Cell adhesion and proliferation}


Cell adhesion and proliferation on the different coatings were determined by cell counting after $1 \mathrm{~h}, 6 \mathrm{~h}$, and 3 days. Substrates were cut into rectangles $(7 \times 15 \mathrm{~mm})$ and placed at the bottom of a 12-well microplate (Iwaki, Tokyo, Japan). The cells were seeded at a density of $1 \times 10^{5}$ cells/well for the cell adhesion assay and $1 \times 10^{4}$ cells/well for the cell proliferation assay. At $1 \mathrm{~h}, 6 \mathrm{~h}$, and 3 days after cell seeding, the wells were washed with phosphate buffered saline (PBS), fixed with $2.5 \%$ glutaraldehyde, and stained with 4',6-diamidino-2-phenylindole (DAPI; Merck, Darmstadt, Germany). The cells were then

observed under a fluorescence microscope (IX81; Olympus, Tokyo, Japan). Ten photographs of each substrate were acquired, and cell numbers were counted using ImageJ $1.43 \mathrm{e}$ software. All experiments were performed with 3 samples.

\subsubsection{Cell morphology}

Cells cultured on each substrate for $1 \mathrm{~h}$ and 3 days were washed with PBS, fixed with $2.5 \%$ glutaraldehyde, dehydrated with increasing concentrations of ethanol $(50 \%, 70 \%$, $80 \%, 90 \%, 95 \%$, and 100\%), and subsequently critical-point dried. The samples were coated with a thin layer of platinum-palladium and observed under a scanning electron microscope (SEM, S-4800; Hitachi, Tokyo, Japan).

\subsubsection{Immunofluorescence staining}

Immunofluorescence staining for F-actin and vinculin was used to investigate cell attachment and morphology. Cells cultured on each substrate for 3 days were washed with 
PBS and fixed with $3.7 \%$ formaldehyde in PBS for $15 \mathrm{~min}$. The cells were subsequently permeabilized with $0.1 \%$ Triton $\mathrm{X}-100$ for $20 \mathrm{~min}$ and blocked with $1 \%$ bovine serum albumin in PBS for $30 \mathrm{~min}$. Vinculin was visualized using a subcellular structure localization kit (Millipore, MA, USA) according to the manufacturer's protocol. Briefly, cells were incubated using a 1:100 dilution of mouse anti-human vinculin for $1 \mathrm{~h}$ at room temperature; the cells were then labeled with a goat anti-mouse IgG FITC-conjugated secondary antibody. For nuclear and F-actin staining, cells were incubated with DAPI (1:1000) and rhodamine-phalloidin $(1: 1000)$, respectively, at $37^{\circ} \mathrm{C}$ in a humidified atmosphere for $45 \mathrm{~min}$. Cells were visualized under a fluorescence microscope (BZ9000; Keyence, Tokyo, Japan).

\subsubsection{Fabrication of the SWCNT-silicone electrode and dielectrophoretic (DEP) patterning} of C2C12 cells with SWCNT-coated silicone

Thin films of SWCNTs $\left(10 \mu \mathrm{g} / \mathrm{cm}^{2}\right)$ were cut into squares and 2 films were positioned leaving a 1-mm gap between them. Silicone elastomer and cross-linker were then mixed, poured onto the film, and polymerized. These silicone-coated squares subsequently formed a face-to-face electrode.

Cells were dispersed in dielectrophoresis buffer (Iworks Co. Ltd., Osaka, Japan) at a concentration of $1 \times 10^{6}$ cells $/ \mathrm{mL}$. The cells were introduced onto the silicone electrode, and DEP was carried out using a multifunction generator (WF-1973; NF Co., Kanagawa, Japan) at $1 \mathrm{MHz}, 8 \mathrm{Vpp} \mathrm{AC}$ voltage for $5 \mathrm{~min}$. 


\subsection{Statistical analysis}

Each data point is expressed as mean \pm SD. Differences was analyzed by ANOVA followed by Tukey's multiple comparison test; $p<0.05$ was considered statistically significant.

\section{Results}

\subsection{Characterization of materials}

Typical AFM images are shown in Figure 2. Uncoated silicone had a relatively smooth topography with several rough spots (Figure 2A). The morphology of the CNT bundles could be clearly observed on each CNT-coated surface. SWCNTs formed long straight bundles distributed over the silicone surface (Figure 2B); meanwhile, MWCNTs on the surface had a relatively tangled and curled structure (Figure 2C). Section analysis showed that the CNT coatings increased the asperity of the surface. The contact angles on uncoated silicone, SWCNT silicone, and MWCNT silicone were $99.1^{\circ} \pm 0.9^{\circ}, 99.5^{\circ} \pm 2.4^{\circ}$, and $96.1^{\circ} \pm 1.8^{\circ}$, respectively; there were no significant differences between groups.

No apparent detachment of CNTs was observed in the tape tests. The optical transparency of the SWCNT-coated silicone before and after the tape test was $50.1 \% \pm$ $1.5 \%$ and $49.9 \% \pm 1.5 \%$, respectively; the transparency of the MWCNT-coated silicone before and after the tape test was $49.1 \% \pm 1.3 \%$ and $49.2 \% \pm 1.4 \%$, respectively. Neither type of CNT-coating resulted in significantly higher transparency after the tape test. AFM 
images after the tape test revealed some changes in surface morphology (Figure 3). The surfaces of both SWCNT- and MWCNT-coated silicone became more uneven compared to the surfaces before the tape test. Although the surface morphology was not clear, CNT bundles were still observed.

Figure 4A shows the sheet resistance of the both types of CNT-coated silicone. The resistance decreased with increasing amounts of CNTs. However, there was a large difference between SWCNT- and MWCNT-coated silicones. The conductivity of $10 \mu \mathrm{g} / \mathrm{cm}^{2}$ SWCNT coating $(0.86 \mathrm{k} \Omega / \mathrm{sq})$ was more than 1,700 fold greater than the same amount of MWCNT coating $\left(1.5 \times 10^{3} \mathrm{k} \Omega / \mathrm{sq}\right)$. Although $1 \mu \mathrm{g} / \mathrm{cm}^{2} \mathrm{SWCNT}$ coating was conductive $\left(4.2 \times 10^{3} \mathrm{k} \Omega / \mathrm{sq}\right)$, silicone coated with small amounts of MWCNTs $\left(1,2.5\right.$, and $\left.5 \mu \mathrm{g} / \mathrm{cm}^{2}\right)$ did not exhibit any conductivity at all (resistance beyond the measurement range); furthermore, even with large amounts $\left(7.5\right.$ and $\left.10 \mu \mathrm{g} / \mathrm{cm}^{2}\right)$, the conductivity was far less than that of the SWCNT coating. At 20\% stretch, the sheet resistance of SWCNT-coated silicone increased approximately 1.5 times. The sheet resistance of the MWCNT-coated silicone also increased with stretching; the resistance of $7.5 \mu \mathrm{g} / \mathrm{cm}^{2}$ MWCNTs increased beyond the measurable range. Figure 4B shows the sheet resistance of SWCNT-coated silicone $\left(10 \mu \mathrm{g} / \mathrm{cm}^{2}\right)$ measured after 100 cycles of uniaxial stretching. The resistance increased 1.5 times relative to the first $20 \%$ stretch but did not change significantly even after 100 cycles of stretching.

\subsection{Cell culture analysis}




\subsubsection{Cell adhesion and proliferation}

To investigate the potential usefulness of CNT-coated silicone as a tissue engineering scaffold, its biocompatibility was evaluated on the basis of the adhesion and proliferation properties of $\mathrm{C} 2 \mathrm{C} 12$ cells. Cells exhibited different attachment speed with different coatings (Figure 5). After $1 \mathrm{~h}$ incubation, MWCNT-coated surfaces showed better cell attachment than SWCNT-coated surfaces. At $6 \mathrm{~h}$, the cell numbers on both surfaces had increased, but more remained on the MWCNT-coated surfaces. Uncoated silicone did not support cell adhesion at either time point. SEM images corroborate the cell adhesion data (Figure 6). Cells were observed on both SWCNT- and MWCNT-coated silicone (Figure 6B, C). In contrast, cells attached very poorly to the untreated surface (Figure 6A).

Figure 7 shows cell proliferation 3 day after seeding. Significantly more cells were present on both CNT-coated surfaces than on uncoated silicone. The SEM images of cells cultured on each substrate at 3 days are shown in Figure 8. Cells seeded on CNT-coated silicone proliferated well and covered most of the surface, while hardly any cells were found on uncoated silicone.

Representative immunofluorescence images of vinculin (a focal adhesion protein) and F-actin are shown in Figure 9. Unlike uncoated silicone, both types of CNT-coated silicone were covered with proliferated cells. Vinculin staining revealed clusters at the

periphery of the cells that were co-localized with the ends of actin fibers. This expression of focal adhesion proteins indicates the existence of cell-substrate adhesion. The vinculin 
clusters could be seen more clearly on MWCNT-coated silicone than on SWCNT-coated silicone (Figure 9, insets).

\subsubsection{DEP with SWCNT-coated silicone.}

A schematic illustration of an SWCNT-coated silicone electrode is shown in Figure 10A. One sheet of silicone was divided into 2 regions: a black SWCNT-coated part and a transparent uncoated part. When an electric field ( $8 \mathrm{Vpp}, 1 \mathrm{MHz})$ was applied, cells dispersed in the buffer immediately attracted between the electrodes and aligned themselves in a pearl-chain pattern within 5 min (Figure 10B). The cell aggregation increased until the voltage was turned off.

\section{Discussion}

The objective of this study was to fabricate a flexible electro-conductive scaffold that can contribute to cellular organization. To create such scaffold, we coated silicone sheet surfaces with CNTs. This coating layer enabled $\mathrm{C} 2 \mathrm{C} 12$ cells to adhere to the silicone sheet and also provided electro-conductivity, which was maintained after cyclic stretching. On the basis of these properties, cell alignment with DEP was performed on the CNT-coated silicone, which acted as a flexible electrode.

In a previous study, we created coated silicone by dispersing CNTs on a prefabricated silicone sheet. However, the CNT coatings were not sufficiently fixed. To solve this problem, we adopted a fabrication method that involved polymerizing silicone 
over a thin film of CNTs. In this method, one side of the CNT film was fixed onto the silicone while the other side remained exposed to the surface (Figure 2B, C). The adhesion of the CNTs to the silicone surfaces was evaluated by the tape test. From the AFM images after the tape test, both SWCNT- and MWCNT-coated surfaces became more uneven, and some prominences were observed (Figure 3). These deformations were probably due to the adhesive force of the tape. However, since CNT bundles were observed after the tape test, most of the CNTs were considered to be fixed to the silicone surface. The optical transparency results indicated that negligible CNT coatings were removed due to the tape test, even though some CNTs on the surface layer may have detached. The AFM images and optical transparency indicate that both types of CNTs were fixed to the silicone surface using this coating method.

The biocompatibility of CNT-coated silicone was evaluated by assessing the adhesion and proliferation of $\mathrm{C} 2 \mathrm{C} 12$ cells (Figures 5-8). The improvements in cell adhesion and proliferation with CNT coatings are probably due to the changes in surface topography. The nanoscale surface topography of artificial materials plays a significant role in modifying cell behavior $[33,34]$. Many studies show that nanotopography affects cellular responses such as adhesion, proliferation, and migration. Kobayashi et al. evaluated cell adhesion and growth on silicone sheets with different degrees of roughness and reported that cell adhesion increases with increasing surface roughness $[35,36]$. In the present study, both types of CNTs formed a net-like structure on the silicone surface (Figure 2B, C) and enhanced cell adhesion. As mentioned in section 1, the diameter of collagen fibrils is 
around 40-100 nm [18,19]; the fibrils consist of collagen molecules $1.5 \mathrm{~nm}$ wide and 300 nm long [17]. The diameters of MWCNTs and SWCNTs in this study were 20-40 and $0.8-2.5 \mathrm{~nm}$, respectively. As seen in Figure 2B, SWCNTs assembled as thick bundles. The geometric similarity of CNTs to collagen fibrils might be responsible for their enhancement of cell adhesion.

Initial attachment to MWCNTs was better than to SWCNTs (Figure 5). Moreover, focal adhesions were observed more clearly on MWCNTs (Figure 9). These differences are probably due to the structural differences between SWCNTs and MWCNTs. The results of the present study are corroborated by our previous results in that vinculin expression was altered and osteoblastic cells strongly adhered to MWCNTs [16]. However, cell proliferation was favorable on both types of CNT coatings, and cell numbers after 3 days were not significantly different (Figure 7). Martínez et al. report that substrate topography directly affects cell attachment and orientation; however, the effects of topography on cell proliferation and differentiation remain unclear [34]. Our results on attachment and proliferation on both types of CNT sheet are concordant with these previously reported results.

The wettability of the 3 different surfaces was evaluated to further investigate cell attachment. However, there were no significant differences in the contact angles between the 3 surfaces. Our previous results also indicate a lack of differences in the contact angles between uncoated silicone and CNT-coated silicone. Thus, in the case of CNT-coated silicone, we consider that the cell adhesion was due to the change in surface structure rather 
than surface energy.

The difference in the electro-conductivity between SWCNT- and MWCNT-coated sheets is also likely due to structural differences between the CNTs. As shown in Figure 2, MWCNTs have a curled, short structure, whereas SWCNTs are long and straight. Electricity probably flows more efficiently through the fine network of SWCNTs; therefore, SWCNT-coated silicone exhibits the highest electro-conductivity. There are several reports on thin conductive film using SWCNTs; conductivity in those reports was $0.2-4.3 \mathrm{k} \Omega / \mathrm{sq}$ [37-40]. The sheet resistance of the SWCNT sheet $\left(10 \mu \mathrm{g} / \mathrm{cm}^{2}\right)$ fabricated in this study was approximately $0.8 \mathrm{k} \Omega / \mathrm{sq}$ (Figure 4A). Even after 100 cycles of stretching, the resistance had increased 1.5 times (Figure 4B) but was still comparable with previously reported values.

To take advantage of the electrical properties of CNTs, DEP was tested as one of the potential applications of CNT scaffolds. Based on the results of the surface conductivity test, $10 \mu \mathrm{g} / \mathrm{cm}^{2}$ SWCNT coating was chosen as the electrode for DEP. The SWCNT electrode pattern was fabricated on 1 flexible silicone sheet using this coating method. Figure 10B shows the sheet 5 min after the application of an $\mathrm{AC}$ electric field. Cells were successfully arranged in a pearl-chain pattern between the SWCNT-coated regions. Ho et al. studied liver cell patterning using DEP and reported radial pearl-chain patterns on planar electrodes [41]. They concluded that this technique can be applied in tissue engineering and drug development. The DEP results of the current present study indicate that the electro-conductivity of SWCNT coating is sufficient for use as an electrode for cell 
patterning.

These results show that CNT coatings on a silicone surface provide both cellular adhesion and electrical conductivity. With these unique properties, cells seeded on CNT-coated scaffolds can be organized using electricity and can be transplanted into the body. Furthermore, silicone exhibits high oxygen permeability [42], which is an advantage when it is implanted. However, in vivo experiments using this scaffold have not been performed yet. This warrants evaluation of the reaction of surrounding tissue after implantation and confirmation of the long-term stability of these coatings before they can be considered for clinical use. After their safety in the human body is confirmed, the widespread application of these kinds of materials, including their use as electrically conductive tissue scaffolds or implantable electrodes, will be possible.

\section{Conclusions}

In this study, scaffolds exhibiting good cell adhesion and high electro-conductivity were developed. Coating with both SWCNTs and MWCNTs enabled cell adhesion to the silicone surface; $\mathrm{C} 2 \mathrm{C} 12$ cells exhibited good proliferation on both types of CNT-coated silicone. SWCNT-coated silicone exhibited the best electro-conductivity, which was maintained even after 100 cycles of stretching. To take advantage of these properties, DEP was tested as a potential application of these scaffolds; using DEP, cells were successfully arranged on the scaffold. These findings indicate that CNT-coated silicone is beneficial for tissue organization and can be considered a useful material for biomedical scaffolds. 


\section{ACKNOWLWDGMENTS}

The analysis of AFM images were carried out with MFP-3D-BIO-J (Asylum Technology Co., LTD., Japan) at the Open Facility, Hokkaido University Sousei Hall. This work has been supported by a fund of "Research Fellowships of the Japan Society for the Promotion of Science for Young Scientists”. 


\section{REFERENCES}

[1] D. Dado, S. Levenberg, Cell-scaffold mechanical interplay within engineered tissue, Semin. Cell. Dev. Biol. 20 (2009) 656-664.

[2] C.P. Barnes, S.A. Sell, E.D. Boland, D.G. Simpson, G.L. Bowlin. Nanofiber technology: Designing the next generation of tissue engineering scaffolds, Adv. Drug. Deliv. Rev. 59 (2007) 1413-1433.

[3] N. Bock, A. Riminucci, C. Dionigi, A. Russo, A. Tampieri, E. Landi, V.A. Goranov, M. Marcacci, V. Dediu, A novel route in bone tissue engineering: Magnetic biomimetic scaffolds, Acta Biomater. 6 (2010) 786-796.

[4] H.J. Chung, T.G. Park, Surface engineered and drug releasing pre-fabricated scaffolds for tissue engineering, Adv. Drug. Deliv. Rev. 59 (2007) 249-262.

[5] D.P. Byrne, D. Lacroix, J.A. Planell, D.J. Kelly, P.J. Prendergast, Simulation of tissue differentiation in a scaffold as a function of porosity, Young's modulus and dissolution rate: Application of mechanobiological models in tissue engineering, Biomaterials 28 (2007) $5544-5554$.

[6] V. Sgobba, D.M. Guldi, Carbon nanotubes-electronic/electrochemical properties and application for nanoelectronics and photonics, Chem. Soc. Rev. 38 (2009) 165-184.

[7] D. Vairavapandian, P. Vichchulada, M.D. Lay, Preparation and modification of carbon nanotubes: Review of recent advances and applications in catalysis and sensing, Anal. Chim. Acta 626 (2008) 119-129.

[8] M. Foldvari, M. Bagonluri, Carbon nanotubes as functional excipients for 
nanomedicines: I. pharmaceutical properties, Nanomed-Nanotechnol. Biol. Med. 4 (2008) $173-182$.

[9] M. Foldvari, M. Bagonluri, Carbon nanotubes as functional excipients for nanomedicines: II. Drug delivery and biocompatibility issues, Nanomed-Nanotechnol. Biol. Med. 4 (2008) 183-200.

[10] Y. Zhang, Y. Bai, B. Yan, Functionalized carbon nanotubes for potential medicinal applications, Drug. Discov. Today 15 (2010) 428-435.

[11] P.A. Tran, L. Zhang, T.J. Webster. Carbon nano fibers and carbon nanotubes in regenerative medicine, Adv. Drug. Deliv. Rev. 61 (2009) 1097-1114.

[12] S.L. Edwards, J.S. Church, J.A. Werkmeister, J.A.M. Ramshaw, Tubular micro-scale multiwalled carbon nanotube-based scaffolds for tissue engineering, Biomaterials 30 (2009) $1725-1731$.

[13] A Abarrategi, M.C. Gutiérrez, C. Moreno-Vicente, M.J. Hortigüela, V. Ramos, J.L. López-Lacomba, M.L. Ferrer, F. del Monte, Multiwall carbon nanotube scaffolds for tissue engineering purposes, Biomaterials 29 (2008) 94-102.

[14] T.I. Chao, S. Xiang, C.S. Chen, W.C. Chin, A.J. Nelson, C. Wang, J. Lu, Carbon nanotubes promote neuron differentiation from human embryonic stem cells, Biochem. Biophys. Res. Commun. 384 (2009) 426-430.

[15] I. Firkowska, E. Godehardt, M. Giersig, Interaction Between Human Osteoblast Cells and Inorganic Two-Dimensional Scaffolds Based on Multiwalled Carbon Nanotubes: A Quantitative AFM Study, Adv. Funct. Mater. 18 (2008) 3765-3771. 
[16] M. Matsuoka, T. Akasaka, T. Totsuka, F. Watari, Strong adhesion of Saos-2 cells to multi-walled carbon nanotubes, Mater. Sci. Eng. B -Solid State Mater. Adv. Technol. 173 (2010) 182-186.

[17] P. Fratzl, Collagen: structure and mechanics, New York, 2008.

[18] M. Tzaphlidou, P. Berillis, Collagen fibril diameter in relation to bone site. A quantitative ultrastructural study, Micron 36 (2005) 703-705.

[19] K.L. Goh, J. Hiller, J.L. Haston, D.F. Holmes, K.E. K.A. Murdoch, J.R. Meakin, T.J. Wess, Analysis of collagen fibril diameter distribution in connective tissues using small-angle X-ray scattering, Biochimica et Biophysica Acta 1722 (2005) 183-188.

[20] H. Kaji, G. Camci-Unal, R. Langer, A. hademhosseinib, Engineering systems for the generation of patterned co-cultures for controlling cell-cell interactions, Biochim. Biophys. Acta 1810 (2011) 239-250.

[21] G.H. Markx, The use of electric fields in tissue engineering, Organogenesis 4 (2008) $11-17$.

[22] G.H. Markx, C.L. Davey, The dielectric properties of biological cells at radiofrequencies: Applications in biotechnology, Enzyme. Microb. Technol. 25 (1999) $161-171$.

[23] M. Suzuki, T. Yasukawa, H. Shiku, T. Matsue, Negative dielectrophoretic patterning with different cell types, Biosens. Bioelectron. 24 (2008) 1043-1047.

[24] A. Sebastian, A.M. Buckle, G.H. Markx, Tissue Engineering With Electric Fields: Immobilization of Mammalian Cells in Multilayer Aggregates Using Dielectrophoresis, 
Biosens. Bioelectron. 98 (2007) 694-700.

[25] A.P. Legrand, G. Marinov, S. Pavlov, M.F. Guidoin, R. Famery, B. Bresson, Z. Zhang and R. Guidoin, Degenerative mineralization in the fibrous capsule of silicone breast implants, J. Mater. Sci.: Mater. Med. 16 (2005) 477-485.

[26] D.H. Jung, B.R. Kim, J.Y. Choi, Y.S. Rho, H.J. Park and W.W. Han, Gross and pathologic analysis of long-term silicone implants inserted into the human body for augmentation rhinoplasty: 221 revision cases, Plast. Reconstr. Surg. 120 (2007) 1997-2003.

[27] A.L. Boch, É. Hermelin, C. Sainte-Rose and S. Sgouros, Mechanical dysfunction of ventriculoperitoneal shunts caused by calcification of the silicone rubber catheter, $J$. Neurosurg. 88 (1998) 975-982.

[28] M. Matsuoka, T. Akasaka, T. Hashimoto, Y. Totsuka, F. Watari, Improvement in cell proliferation on silicone rubber by carbon nanotube coating, Bio-Med. Mater. Eng. 19 (2009) 155-162.

[29] Y. Ando, X Zhao, Synthesis of Carbon Nanotubes by Arc-Discharge Method, New Diam. Front. Carbon Technol, 16 (2006) 123-137.

[30] Y. Sato, A. Yokoyama, K. Shibata, Y. Akimoto, S. Ogino, Y. Nodasaka, T. Kohgo, K. Tamura, T. Akasaka, M. Uo, K. Motomiya, B. Jeyadevan, M. Ishiguro, R. Hatakeyama, F. Watari, K. Tohji, Influence of length on cytotoxicity of multi-walled carbon nanotubes against human acute monocytic leukemia cell line THP-1 in vitro and subcutaneous tissue of rats in vivo, Mol. BioSyst. 1 (2005) 176-182.

[31] A.F. Stalder, G. Kulik, D. Sage, L. Barbieri and P. Hoffmann, A snake-based approach 
to accurate determination of both contact points and contact angles, Colloids Surf. A 286 (2006) 92-103.

[32] M. Yamashita, M. Agu, Geometrical correction factor for semiconductor resistivity measurements by four-point probe method, Jpn. J. Appl. Phys. 23 (1984) 1499-1504.

[33] C.J. Bettinger, R. Langer, J.T. Borenstein, Engineering Substrate Topography at the Micro- and Nanoscale to Control Cell Function, Angew. Chem. Int. Ed. 48 (2009) $5406-5415$.

[34] M.S. Lord, M. Foss, F. Besenbacher, Influence of nanoscale surface topography on protein adsorption and cellular response, Nano Today 5 (2010) 66-78.

[35] T. Kobayashi, R. Katou, T. Yokota, Y. Suzuki, M. Iwaki, T. Terai, Surface modification of silicone sheets and tubes using plasma-based ion implantation, Surf. Coat. Technol. 201 (2007) 8039-8042.

[36] T. Kobayashi, T. Yokota, R. Kato, Y. Suzuki, M. waki, T. Terai, N. Takahashi, T. Miyasato, H Ujiie, Surface modification of silicone medical materials by plasma-based ion implantation, Nucl. Instrum. Methods Phys. Res. Sect. B -Beam Interact. Mater. Atoms 257 (2007) 128-131.

[37] E. Martínez, E. Engel, J.A. Planell, J. Samitier, Effects of artificial micro- and nano-structured surfaces on cell behavior, Ann. Anat. 191 (2009) 126-135.

[38] T. Kitano, Y Maeda, T Akasaka, Preparation of transparent and conductive thin films of carbon nanotubes using a spreading/coating technique, Carbon 47 (2009) 3559-3565.

[39] M.J. Kim, D.W. Shin, J.Y. Kim, S.H. ark, I.T. Han, J.B. Yoo, The production of a 
flexible electroluminescent device on polyethylene terephthalate films using transparent conducting carbon nanotube electrode, Carbon 47 (2009) 3461-3465.

[40] A. Denneulin, J. Bras, F. Carcone, C. Neuman, A. Blayo, Impact of ink formulation on carbon nanotube network organization within inkjet printed conductive films. Cabon 49 (2011) 2603-2614.

[41] C.T. Ho, R.Z. Lin, W.Y. Chang, H.Y. Chang, C.H. Liu, Rapid heterogeneous liver-cell on-chip patterning via the enhanced field-induced dielectrophoresis trap, Lab. Chip. 6 (2006) 724-734.

[42] M. Nishikawa, T. Yamamoto, N. Kojima, K. Kikuo, T. Fujii, Y. Sakai, Stable Immobilization of Rat Hepatocytes as Hemispheroids Onto Collagen-Conjugated Poly-Dimethylsiloxane (PDMS) Surfaces: Importance of Direct Oxygenation Through PDMS for Both Formation and Function, Biotechnol. Bioeng. 99 (2008) 1472-1481. 
Figure Legends

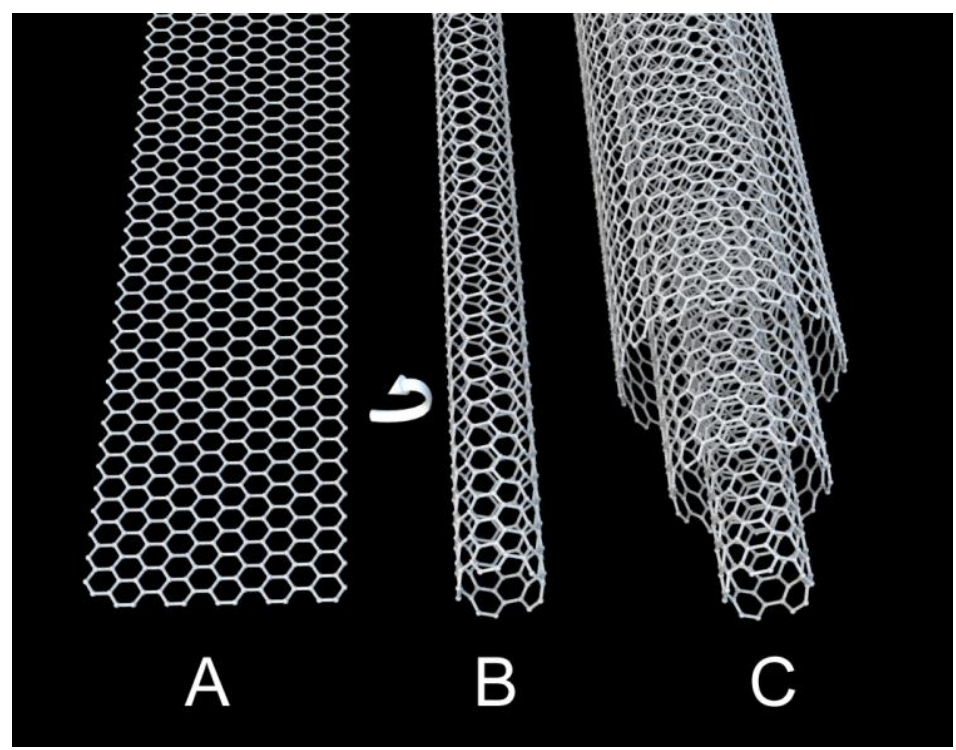

Figure 1 Illustrations of the structure of a (A) graphene sheet, (B) SWCNT, and (C) MWCNT.
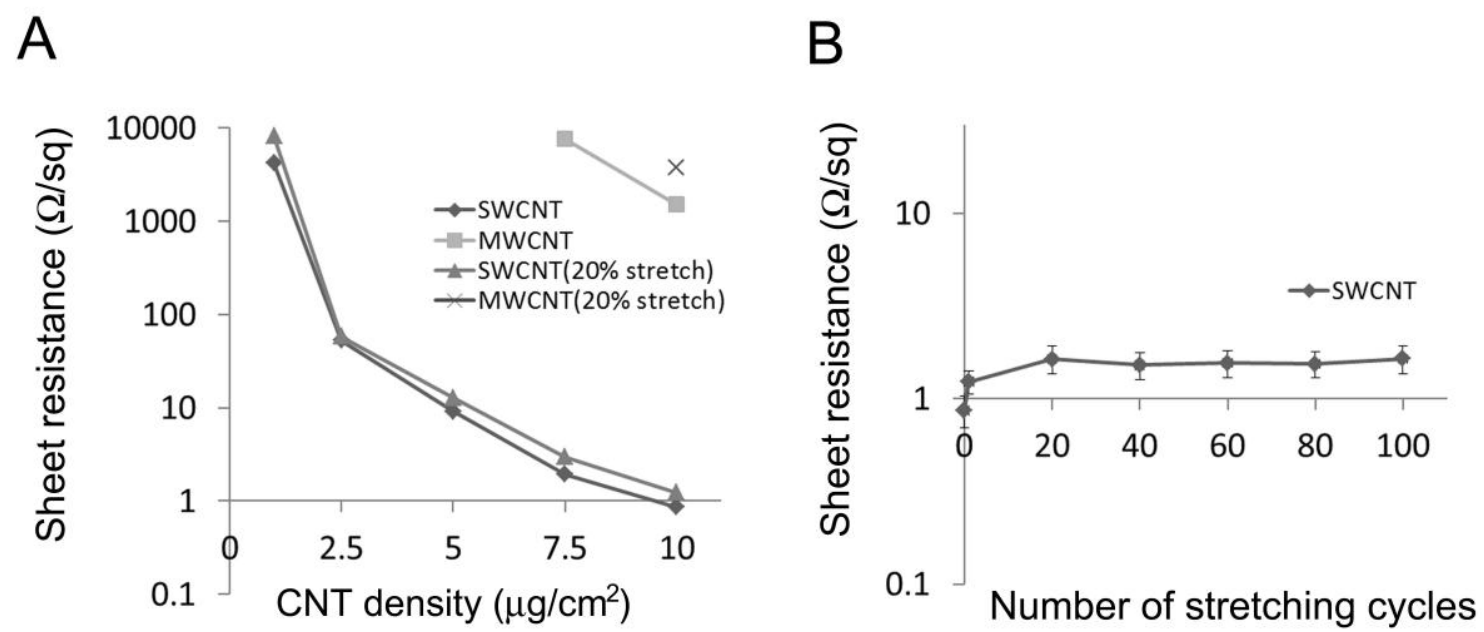

Figure 2 Typical AFM images and section analyses of differently coated silicone surfaces:

(A) Uncoated silicone, (B) SWCNT-coated silicone, and (C) MWCNT-coated silicone. 

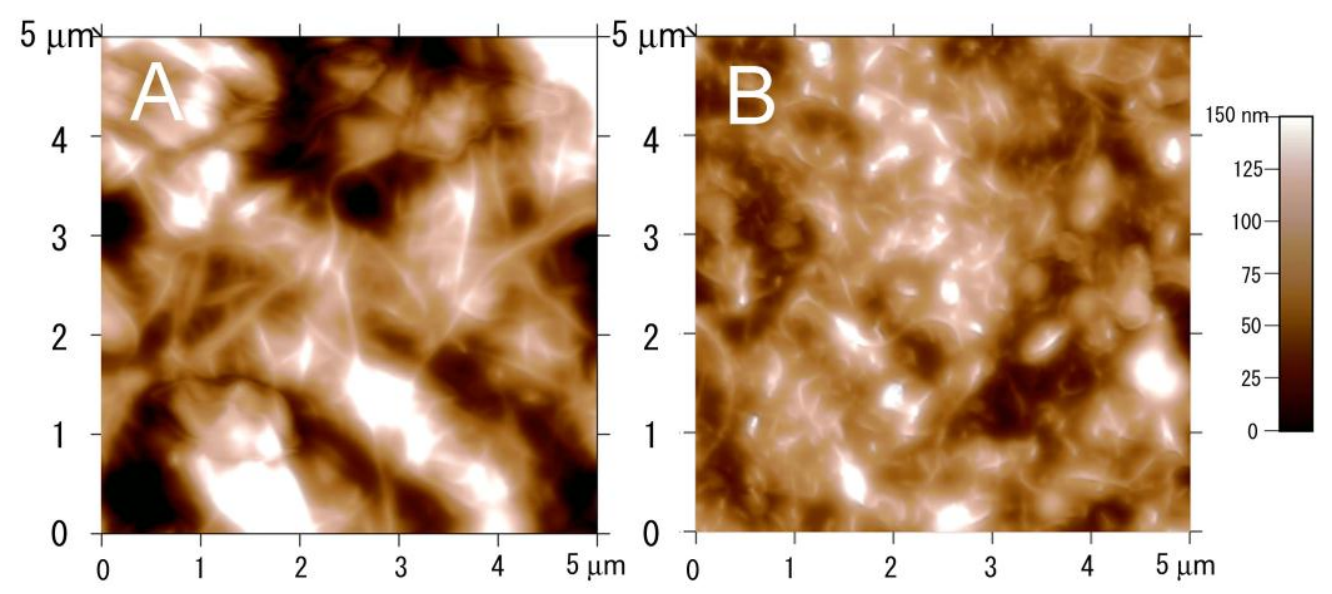

Figure 3 AFM images after the tape-peeling test. (A) SWCNT-coated silicone and (B) MWCNT-coated silicone.
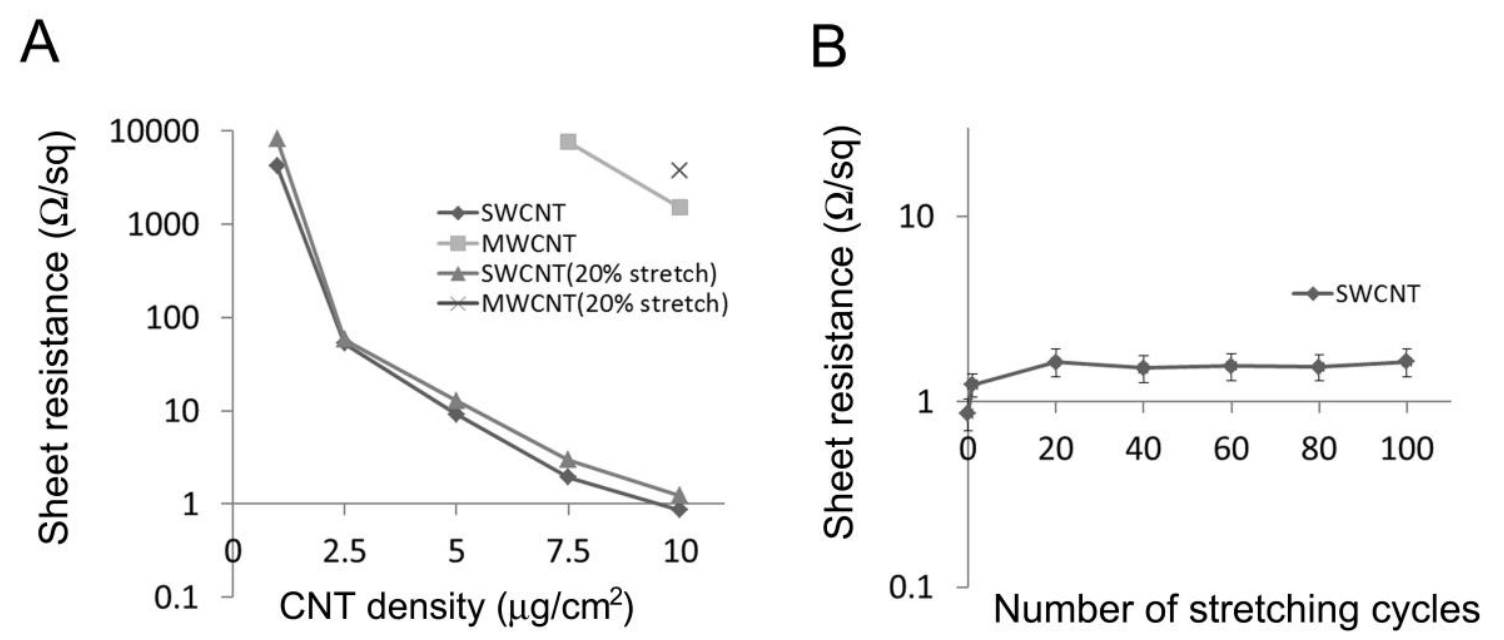

Figure 4 (A) Sheet resistance of both types of CNT-coated silicone, and resistance of $20 \%$ stretched silicone. (B) The sheet resistance of SWCNT-coated silicone $\left(10 \mu \mathrm{g} / \mathrm{cm}^{2}\right)$ was measured after $0,1,20,40,60,80$ and 100 cycles of uniaxial stretch. All measurements were performed with 12 samples. 


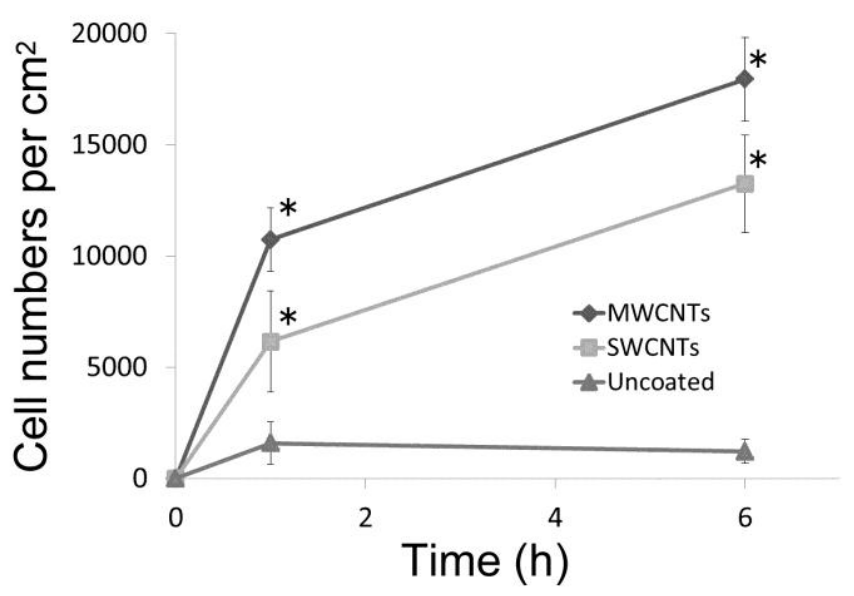

Figure 5 Cell numbers after 1-h and 6-h incubation. Data are expressed as the mean \pm SD (n $=3)$. Values were compared to uncoated silicone. ${ }^{*}$ Statistically significant $(p<0.05)$.
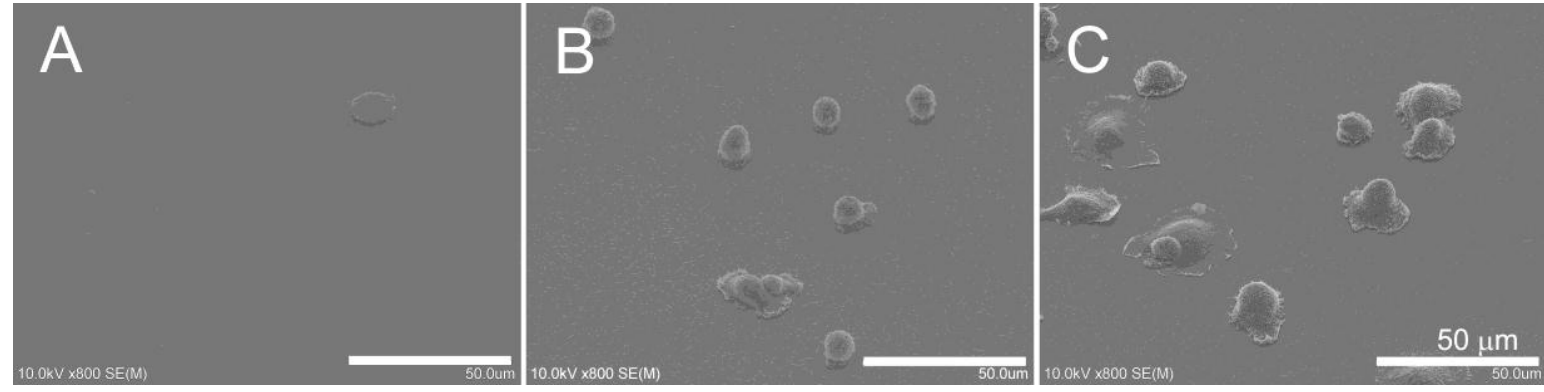

Figure 6 SEM images after $1 \mathrm{~h}$ incubation on differently coated substrate: (A) Uncoated, (B) SWCNTs, and (C) MWCNTs. 


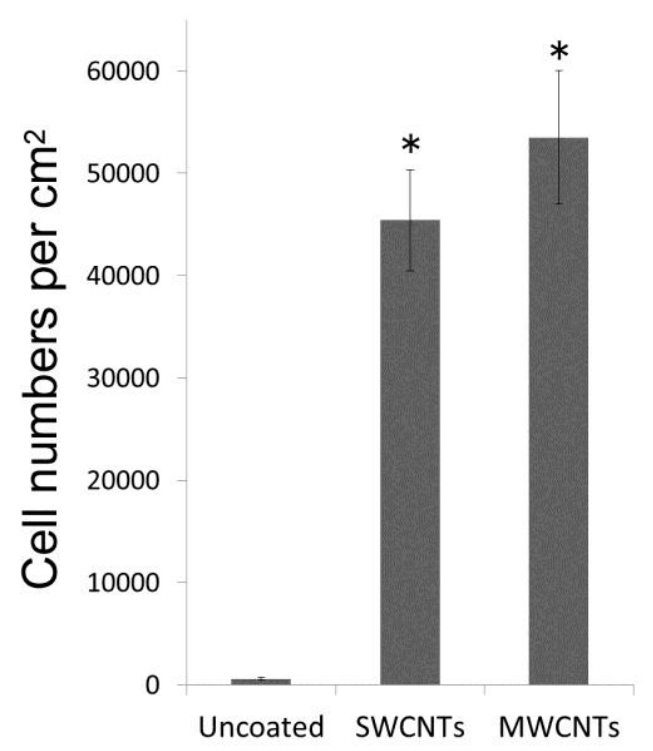

Figure 7 Cell numbers after 3-day incubation. Data are expressed as the mean \pm SD $(n=3)$.

Values were compared to uncoated silicone. ${ }^{*}$ Statistically significant $(p<0.05)$.

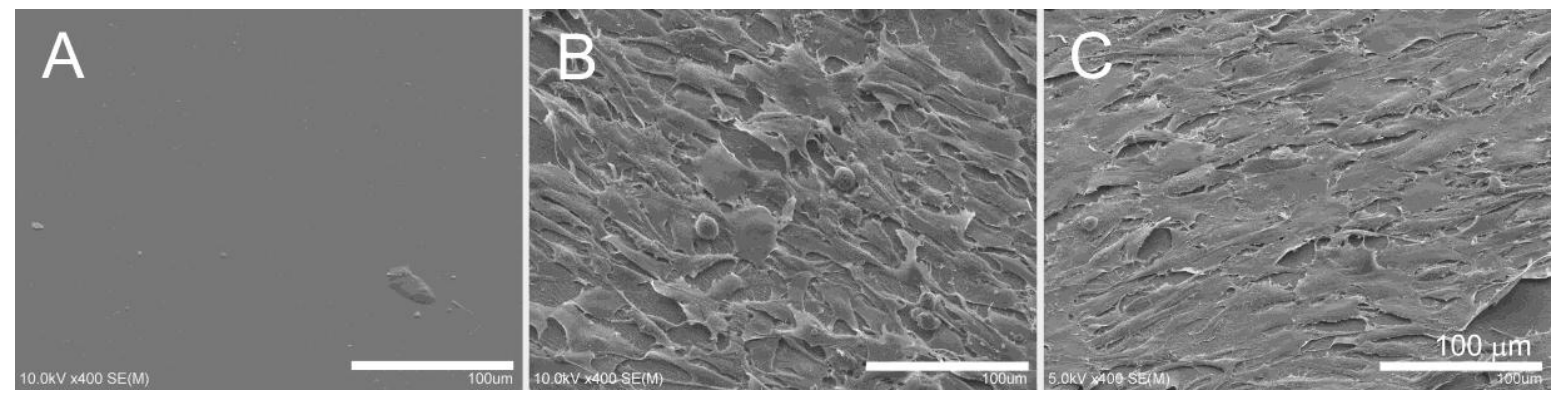

Figure 8 SEM images after 3-day cultivation on differently coated substrate: (A) Uncoated, (B) SWCNTs, and (C) MWCNTs. 


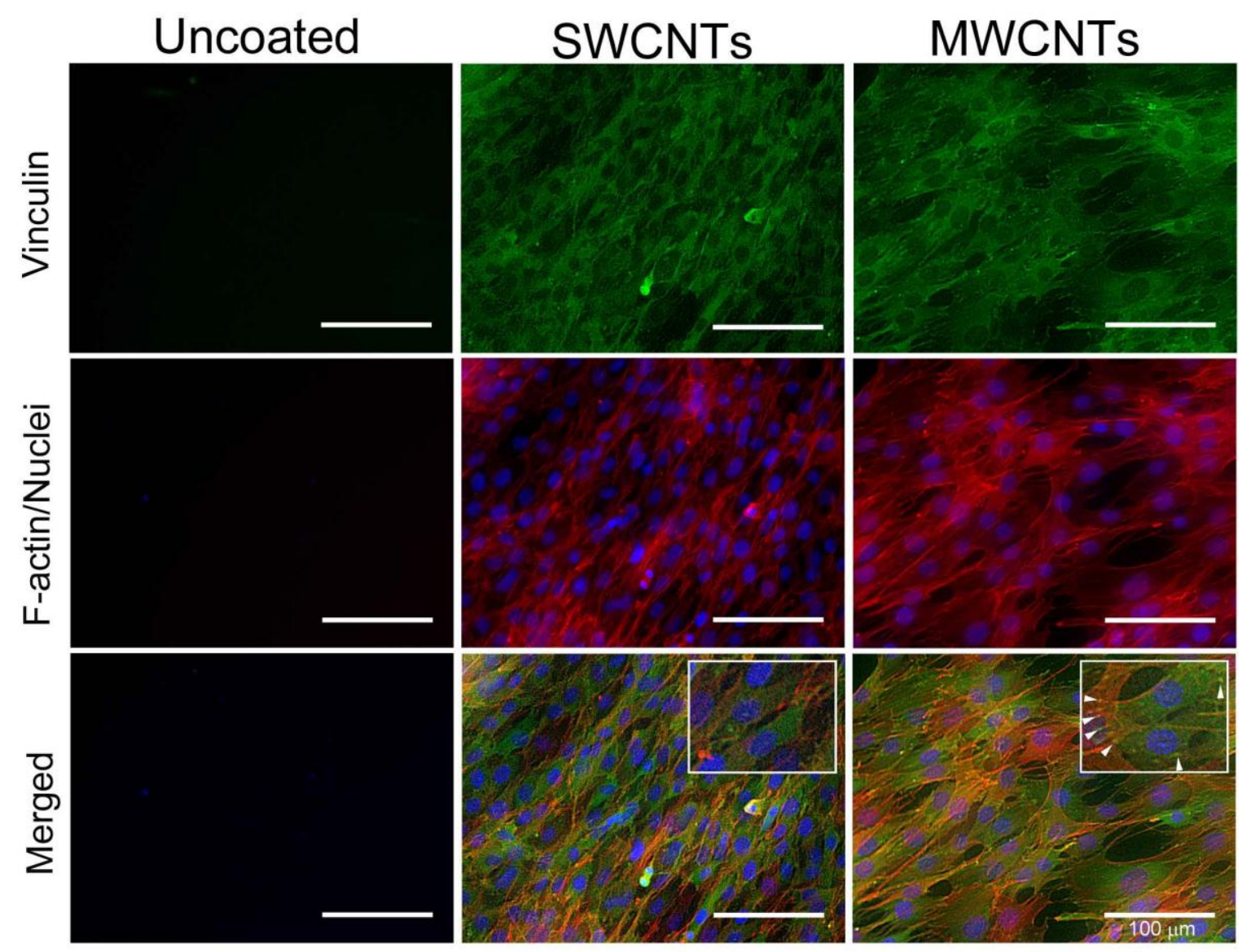

Figure 9 Representative immunofluorescence images of $\mathrm{C} 2 \mathrm{C} 12$ cells on the differently coated substrates after 3 day. Cells were stained for vinculin (green), F-actin (red), and nuclei (blue). Insets show magnified images, and arrows indicate focal contacts. 

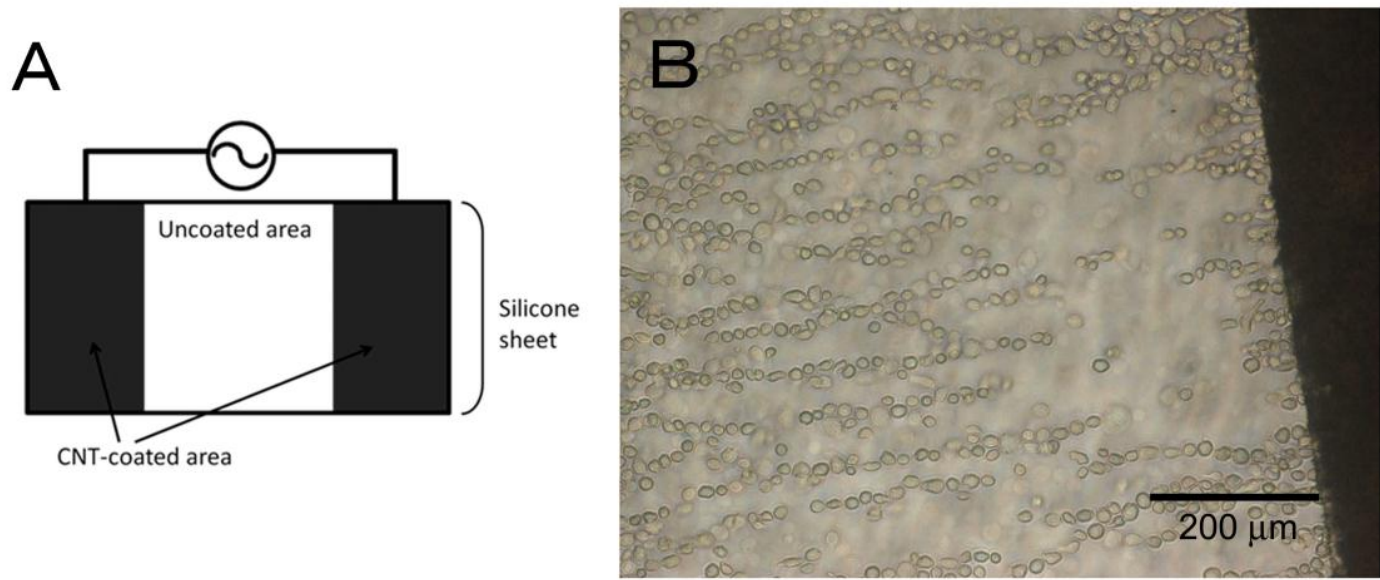

Figure 10 (A) Schematic illustration of the silicone electrode. A silicone sheet was divided into a CNT-coated area (both ends) and an uncoated area. (B) Optical microscope image of C2C12 cells after application of an AC electric field. The cells were aligned between the CNT electrodes. The black area to the right is the SWCNT-coated region and the neighboring transparent area is the uncoated region. 